\title{
Two-particle scattering on the lattice: Phase shifts, spin-orbit coupling, and mixing angles
}

\author{
Buḡra Borasoy $^{a}$, Evgeny Epelbaum ${ }^{a, b}$, Hermann Krebs $^{a, b}$, Dean Lee ${ }^{c, a}$, Ulf-G. Meißner ${ }^{a, b}$ \\ ${ }^{a}$ Helmholtz-Institut für Strahlen- und Kernphysik (Theorie) Universität Bonn, \\ Nußallee 14-16, D-53115 Bonn, Germany \\ ${ }^{b}$ Institut für Kernphysik (Theorie), Forschungszentrum Jülich, D-52425 Jülich, Germany \\ ${ }^{c}$ Department of Physics, North Carolina State University, Raleigh, NC 27695, USA
}

\begin{abstract}
We determine two-particle scattering phase shifts and mixing angles for quantum theories defined with lattice regularization. The method is suitable for any nonrelativistic effective theory of point particles on the lattice. In the center-of-mass frame of the two-particle system we impose a hard spherical wall at some fixed large radius. For channels without partial-wave mixing the partial-wave phase shifts are determined from the energies of the nearly-spherical standing waves. For channels with partial-wave mixing further information is extracted by decomposing the standing wave at the wall boundary into spherical harmonics, and we solve coupled-channels equations to extract the phase shifts and mixing angles. The method is illustrated and tested by computing phase shifts and mixing angles on the lattice for spin-1/2 particles with an attractive Gaussian potential containing both central and tensor force parts.
\end{abstract}




\section{INTRODUCTION}

There have been several recent studies on the subject of lattice simulations for low-energy nuclear physics using effective interactions [1, 2, 3, 4, 5, 6, 7, 8, 9, 10, 11, 12, 13, 14]. Similar lattice effective field theory techniques have been used to study cold atomic Fermi systems in the limit of short-range interactions and large scattering length [15, 16, 17, 18, 19, 20, 21]. In nearly all cases the connection between lattice interactions and physical observables is made using some variant of Lüscher's result [22, 23, 24] relating the energy levels of twobody states in a finite-volume cubic box with periodic boundaries to the infinite-volume scattering matrix.

Lüscher's method has been extended in a number of different ways. Several studies have looked at asymmetric boxes [25, 26], while another considered small volumes where the lattice length $L$ is smaller than the scattering length [27]. There have also been studies of moving frames [28, 29], winding of the interaction around the periodic boundary [30], modifications at finite lattice spacing [8], and techniques to distinguish shallow bound states from scattering states using Levinson's theorem [31]. A very recent study derived finite volume formulas for systems of $n$ bosons with short-range repulsive interactions [32].

While Lüscher's method has been very useful at low momenta, there is currently no technique which is able to determine phase shifts on the lattice at higher energies and higher orbital angular momenta. There is also no technique which can accurately measure spinorbit coupling and partial-wave mixing on the lattice. The physics of spin-orbit coupling and partial-wave mixing is difficult to extract using Lüscher's method due to artifacts generated by the periodic cubic boundary. An example of this problem is shown in the summary and discussion section of this paper. Any method overcoming these theoretical problems would probably not be numerically practical when applied to lattice simulations of quantum chromodynamics. This is because each individual hadron must be constructed as a bound state of quark and gluon fields, and the Monte Carlo signal for hadron-hadron scattering states well above threshold would be very weak. However for an effective lattice theory of fundamental point particles, such a technique should be numerically viable since the full two-particle spectrum is relatively easy to compute.

In this paper we discuss a simple method which directly measures two-particle phase shifts and mixing angles on the lattice. In the center-of-mass frame of the two-particle system 
we impose a hard spherical wall boundary condition at some large radius. Phase shifts and mixing angles are determined from properties of the nearly-spherical standing waves produced by the wall boundary. The method is suitable for any nonrelativistic effective theory of point particles on the lattice with or without spin. We test the method on the lattice using an attractive Gaussian potential for spin-1/2 particles containing both central and tensor force parts.

The organization of the paper is as follows. We first discuss representations of the cubic rotational group. We then introduce the test potential and solve the $S=0$ channels and uncoupled $S=1$ channels up to $J=4$ using the spherical wall method. We then discuss coupled partial waves with a common nodal constraint and the coupled equations needed to solve for the $L=J-1$ and $J+1$ phase shifts and mixing angle for total angular momentum $J$. Finally we solve the coupled $S=1$ channels on the lattice up to $J=4$ and discuss applications of the technique.

\section{CUBIC ROTATIONAL GROUP}

Our choice of lattice regularization reduces the $\mathrm{SO}(3)$ rotational symmetry of continuous space to the cubic rotational group $\mathrm{SO}(3, Z)$. This consists of 24 group elements generated by products of $\pi / 2$ rotations about the $x, y, z$ axes. Since $\mathrm{SO}(3, Z)$ is discrete, we cannot define angular momentum operators $J_{x}, J_{y}, J_{z}$ in the usual sense. However if $R_{\hat{z}}(\pi / 2)$ is the group element for a $\pi / 2$ rotation about the $z$ axis, we can use the $\mathrm{SO}(3)$ relation

$$
R_{\hat{z}}(\pi / 2)=\exp \left[-i \frac{\pi}{2} J_{z}\right]
$$

to define $J_{z}$. The eigenvalues of $J_{z}$ are integers modulo $4 . \quad J_{x}$ and $J_{y}$ can be defined in the same manner.

There are five irreducible representations of the cubic rotational group. These are usually written as $A_{1}, T_{1}, E, T_{2}$, and $A_{2}$. Some of their properties and examples in terms of spherical

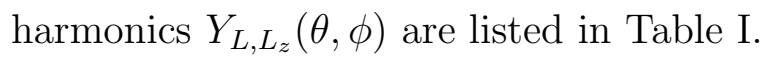

The $2 J+1$ elements of the total angular momentum $J$ representation of $\mathrm{SO}(3)$ break up into smaller pieces consisting of the five irreducible representations. Examples for $J \leq 7$ are shown in Table [11 [33].

In this analysis we consider the scattering of two identical particles and assume that 
TABLE I: Irreducible $\mathrm{SO}(3, Z)$ representations

\begin{tabular}{||c|c|c||}
\hline \hline Representation & $J_{z}$ & Example \\
\hline$A_{1}$ & $0 \bmod 4$ & $Y_{0,0}$ \\
\hline$T_{1}$ & $0,1,3 \bmod 4$ & $\left\{Y_{1,0}, Y_{1,1}, Y_{1,-1}\right\}$ \\
\hline$E$ & $0,2 \bmod 4$ & $\left\{Y_{2,0}, \frac{Y_{2,-2}+Y_{2,2}}{\sqrt{2}}\right\}$ \\
\hline$T_{2}$ & $1,2,3 \bmod 4$ & $\left\{Y_{2,1} \frac{Y_{2,-2}-Y_{2,2}}{\sqrt{2}}, Y_{2,-1}\right\}$ \\
\hline$A_{2}$ & $2 \bmod 4$ & $\frac{Y_{3,2}-Y_{3,-2}}{\sqrt{2}}$ \\
\hline \hline
\end{tabular}

TABLE II: $\mathrm{SO}(3, Z)$ decompositions for $J \leq 7$

\begin{tabular}{||c|c||}
\hline \hline $\mathrm{SO}(3)$ & $\mathrm{SO}(3, Z)$ \\
\hline$J=0$ & $A_{1}$ \\
\hline$J=1$ & $T_{1}$ \\
\hline$J=2$ & $E \oplus T_{2}$ \\
\hline$J=3$ & $T_{1} \oplus T_{2} \oplus A_{2}$ \\
\hline$J=4$ & $A_{1} \oplus T_{1} \oplus E \oplus T_{2}$ \\
\hline$J=5$ & $T_{1} \oplus T_{1} \oplus E \oplus T_{2}$ \\
\hline$J=6$ & $A_{1} \oplus T_{1} \oplus E \oplus T_{2} \oplus T_{2} \oplus A_{2}$ \\
\hline$J=7$ & $T_{1} \oplus T_{1} \oplus E \oplus T_{2} \oplus T_{2} \oplus A_{2}$ \\
\hline \hline
\end{tabular}

the interactions are parity conserving. Therefore in continuous space the two particles share the same group representation for intrinsic spin and the same group representation for all other internal quantum symmetries. As a result each two-particle state must be symmetric or antisymmetric under parity and symmetric or antisymmetric with respect to internal symmetry quantum numbers. Once these are fixed the overall Fermi or Bose statistics determines whether the total intrinsic spin combination must be symmetric or antisymmetric.

There are well-known problems associated with massless fermions on the lattice. However for nonrelativistic particles one can easily find a lattice Hamiltonian or Euclidean action which maintains exact parity invariance and all internal symmetries. Therefore the symmetry or antisymmetry of intrinsic spin on the lattice is unambiguous and the same as in continuous space. For two spin-1/2 particles where the total intrinsic spin is $S=0$ or 
$S=1$, this is enough to specify the intrinsic spin representation completely. If the intrinsic spin is symmetric then the $\mathrm{SO}(3, Z)$ representation is $A_{1}$. If the intrinsic spin is antisymmetric then the $\mathrm{SO}(3, Z)$ representation is $T_{1}$. These are in one-to-one correspondence with the representations $S=0$ and $S=1$ in continuous space, respectively, and so there is no confusion in borrowing the continuous space names for the two cases. For particles with higher intrinsic spin, there is in general some unphysical mixing on the lattice among even values of $S$ and among odd values of $S$. The same mixing on the lattice occurs among even values and odd values of orbital angular momentum $L$ regardless of the intrinsic spin.

\section{TEST POTENTIAL}

There is an endless variety of different interactions one can study on the lattice. For our analysis we choose a simple pedagogical example for which the continuum limit is relatively easy to compute. We choose a bounded short-range potential producing both a central force and tensor force. This yields all of the essential complications of spin-orbit coupling with partial-wave mixing while leaving out additional issues of ultraviolet divergences and singular interactions. We take a system of identical spin- $1 / 2$ particles with mass $m$ and a spin-dependent potential,

$$
V(\vec{r})=C\left\{1+\frac{r^{2}}{R_{0}^{2}}\left[3\left(\hat{r} \cdot \vec{\sigma}_{1}\right)\left(\hat{r} \cdot \vec{\sigma}_{2}\right)-\vec{\sigma}_{1} \cdot \vec{\sigma}_{2}\right]\right\} \exp \left(-\frac{1}{2} \frac{r^{2}}{R_{0}^{2}}\right) .
$$

The range of the potential is set by the parameter $R_{0}$. We leave the internal symmetry group unspecified and consider all possible combinations of symmetry or antisymmetry for parity and intrinsic spin.

The tensor operator

$$
S_{12}(\hat{r})=3\left(\hat{r} \cdot \vec{\sigma}_{1}\right)\left(\hat{r} \cdot \vec{\sigma}_{2}\right)-\vec{\sigma}_{1} \cdot \vec{\sigma}_{2}
$$

is of considerable general interest in physics. It is produced both by one-pion exchange in low-energy nuclear physics as well as by magnetic and electric dipole interactions in atoms and molecules. The parameters $C, R_{0}$, and $m$ we choose are motivated by low-energy 
nuclear physics,

$$
\begin{aligned}
C & =-2 \mathrm{MeV} \\
R_{0} & =2 \times 10^{-2} \mathrm{MeV}^{-1} \\
m & =938.92 \mathrm{MeV}
\end{aligned}
$$

This produces a very shallow bound state in the ${ }^{3} S(D)_{1}$ channel with energy $-0.155 \mathrm{MeV}$. The parenthesis in our spectroscopic notation indicates a mixture of two partial waves. For example ${ }^{3} S(D)_{1}$ indicates a mixture of $S$ and $D$ partial waves. The ordering of the partial waves, $S(D)$ rather than $D(S)$, indicates that this state becomes a pure $S$ wave when the tensor part of the interaction is continuously dialed down to zero. This identification is unambiguous in finite volume systems where the energy levels are discrete.

For total intrinsic spin $S=0$ the tensor operator $S_{12}$ vanishes. And so for this case it suffices to consider just the central part of the potential,

$$
V_{0}(\vec{r})=C \exp \left(-\frac{1}{2} \frac{r^{2}}{R_{0}^{2}}\right),
$$

for particles with no intrinsic spin at all. We start first with this simplest case. Afterwards we consider $S=1$ for uncoupled channels and then finally $S=1$ in coupled channels.

\section{INTRINSIC SPIN $S=0$}

We measure phase shifts by imposing a hard spherical wall boundary on the relative separation between the two particles at some chosen radius $R_{\text {wall }}$. Viewed in the center-ofmass frame we solve the Schrödinger equation for spherical standing waves which vanish at $r=R_{\text {wall }}$. This is sketched in Fig. 1, We are of course assuming that the original lattice system is large enough to hold a sphere of radius $R_{\text {wall }}$. The reason for this spherical wall is to remove copies of the two-particle interactions due to the periodic boundaries of the lattice. It is this feature that distinguishes our method from Lüscher's method [24].

For values of $r$ beyond the range of the interaction, the spherical standing wave in continuous space can be decomposed as a superposition of products of spherical harmonics and spherical Bessel functions,

$$
\left[\cos \delta_{L} \times j_{L}(k r)-\sin \delta_{L} \times y_{L}(k r)\right] Y_{L, L_{z}}(\theta, \phi)
$$




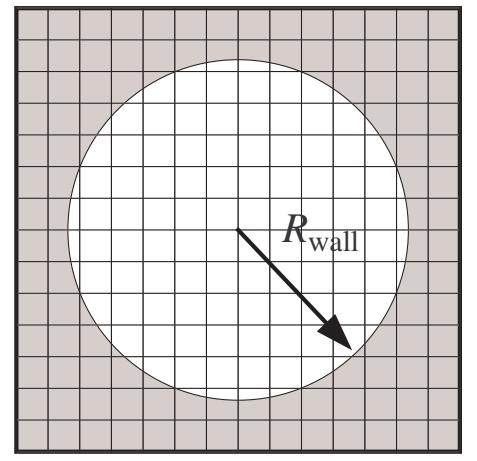

FIG. 1: Spherical wall imposed in the center-of-mass frame.

where the center-of-mass energy of the spherical wave is

$$
E=2 \times \frac{k^{2}}{2 m}=\frac{k^{2}}{m}
$$

and the phase shift for partial wave $L$ is $\delta_{L}$. Therefore we know $k$ from the energy $E$, and the phase shift $\delta_{L}$ is determined by setting the wavefunction (8) equal to zero at the wall boundary,

$$
\begin{gathered}
\cos \delta_{L} \times j_{L}\left(k R_{\mathrm{wall}}\right)=\sin \delta_{L} \times y_{L}\left(k R_{\mathrm{wall}}\right), \\
\delta_{L}=\tan ^{-1}\left[\frac{j_{L}\left(k R_{\mathrm{wall}}\right)}{y_{L}\left(k R_{\text {wall }}\right)}\right] .
\end{gathered}
$$

On the lattice there is some ambiguity on the precise value of $R_{\text {wall }}$ since the components of $\vec{r}$ must be integer multiples of the lattice spacing. We resolve this ambiguity by finetuning the value of $R_{\text {wall }}$ for each standing wave so that $\delta_{L}$ equals zero when the particles are non-interacting. This is illustrated in the following discussion of the lattice calculation.

\section{LATTICE CALCULATION FOR $S=0$}

Since our method is intended as a tool for simulations of few- and many-body systems using lattice effective field theory, we present the analysis using the standard formalism of lattice field theory developed in the literature. For the free part of the lattice action we use the same lattice action defined in [13] with spatial lattice spacing $a=(100 \mathrm{MeV})^{-1}$ and temporal lattice spacing $a_{t}=(70 \mathrm{MeV})^{-1}$. We define $\alpha_{t}$ as the ratio between lattice spacings, $\alpha_{t}=a_{t} / a$. Throughout we use dimensionless parameters and operators, which 
correspond with physical values multiplied by the appropriate power of $a$. Final results are presented in physical units with the corresponding unit stated explicitly.

Since the temporal lattice spacing is nonzero we work with transfer matrices rather than the Hamiltonian directly. In simple terms the transfer matrix is just the exponential of the Hamiltonian $\exp (-H \Delta t)$, where $\Delta t$ equals one temporal lattice spacing. More precisely the free-particle transfer matrix is defined as

$$
M_{\text {free }} \equiv: \exp \left(-H_{\text {free }} \alpha_{t}\right):
$$

where the :: symbols indicate normal ordering. We use the $O\left(a^{4}\right)$-improved free lattice Hamiltonian,

$$
\begin{aligned}
H_{\text {free }} & =\frac{49}{12 m} \sum_{\vec{n}} \sum_{j=\uparrow, \downarrow} a_{j}^{\dagger}(\vec{n}) a_{j}(\vec{n}) \\
& -\frac{3}{4 m} \sum_{\vec{n}} \sum_{j=\uparrow, \downarrow} \sum_{l=1,2,3}\left[a_{j}^{\dagger}(\vec{n}) a_{j}(\vec{n}+\hat{l})+a_{j}^{\dagger}(\vec{n}) a_{j}(\vec{n}-\hat{l})\right] \\
& +\frac{3}{40 m} \sum_{\vec{n}} \sum_{j=\uparrow, \downarrow} \sum_{l=1,2,3}\left[a_{j}^{\dagger}(\vec{n}) a_{j}(\vec{n}+2 \hat{l})+a_{j}^{\dagger}(\vec{n}) a_{j}(\vec{n}-2 \hat{l})\right] \\
& -\frac{1}{180 m} \sum_{\vec{n}} \sum_{j=\uparrow, \downarrow} \sum_{l=1,2,3}\left[a_{j}^{\dagger}(\vec{n}) a_{j}(\vec{n}+3 \hat{l})+a_{j}^{\dagger}(\vec{n}) a_{j}(\vec{n}-3 \hat{l})\right] .
\end{aligned}
$$

The vector $\vec{n}$ denotes integer-valued coordinate vectors on a spatial three-dimensional lattice, and $\hat{l}=\hat{1}, \hat{2}, \hat{3}$ are lattice unit vectors in each of the spatial directions.

Since the potential energy is finite we can take the lattice potential at $\vec{n}$ to agree with the continuum potential at $\vec{r}=\vec{n} a$. For $S=0$ we can omit the tensor part of the potential, and so the transfer matrix is

$$
M \equiv: \exp \left[-H_{\text {free }} \alpha_{t}-\frac{\alpha_{t}}{2} \sum_{\vec{n}_{1}, \vec{n}_{2}} V_{0}\left(\vec{n}_{1}-\vec{n}_{2}\right) \rho^{a^{\dagger}, a}\left(\vec{n}_{1}\right) \rho^{a^{\dagger}, a}\left(\vec{n}_{2}\right)\right]:
$$

where $\rho^{a^{\dagger}, a}(\vec{n})$ is the particle density operator

$$
\rho^{a^{\dagger}, a}(\vec{n})=\sum_{j=\uparrow, \downarrow} a_{j}^{\dagger}(\vec{n}) a_{j}(\vec{n})
$$

We calculate eigenvalues of the transfer matrix using the Lanczos method [34]. In the transfer matrix formalism eigenvalues of the transfer matrix are interpreted as exponentials of the energy,

$$
M|\Psi\rangle=\lambda|\Psi\rangle=e^{-E \alpha_{t}}|\Psi\rangle .
$$



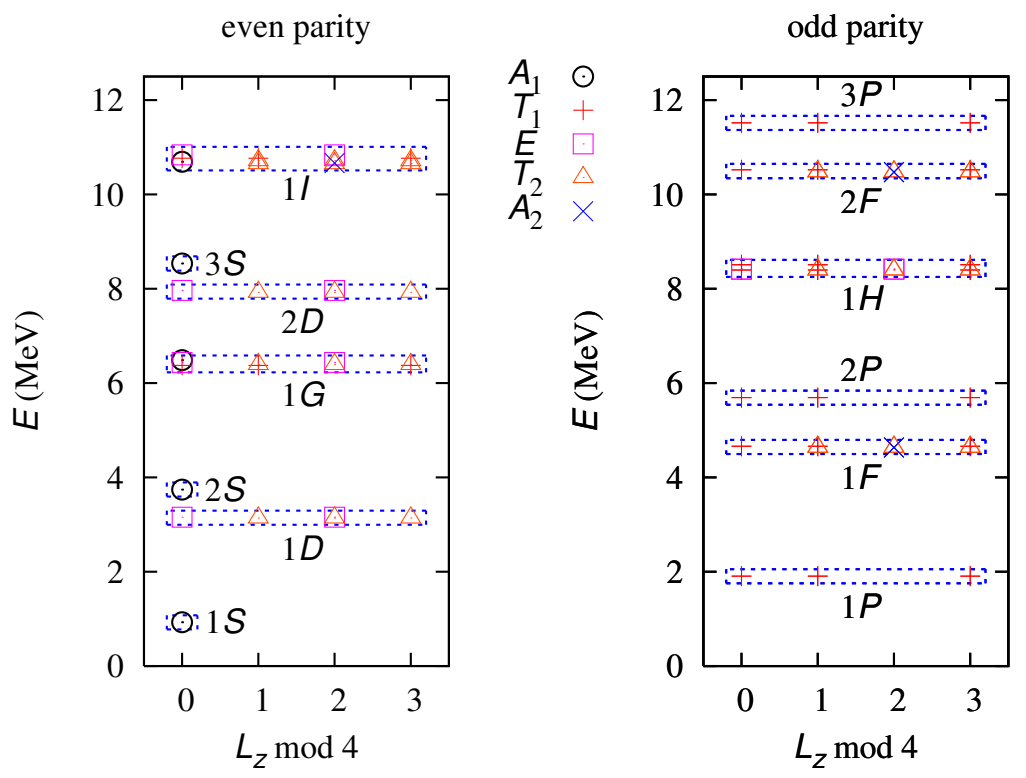

FIG. 2: Free particle spectrum for standing waves with radius $R_{\text {wall }}=10+\epsilon$ lattice units.

In Fig. 2 we show the free two-particle energy spectrum in the center-of-mass frame. We have set the spherical wall so that the amplitude for two-particle separation greater than 10 lattice units is strongly suppressed by a large potential energy due to the spherical wall. We can write this as

$$
V_{0}\left(\vec{n}_{1}-\vec{n}_{2}\right) \rightarrow V_{0}\left(\vec{n}_{1}-\vec{n}_{2}\right)+V_{\text {wall }} \times \theta\left(\left|\vec{n}_{1}-\vec{n}_{2}\right|-(10+\epsilon)\right),
$$

where $\theta$ is the unit step function and $\epsilon$ is a small positive number. This choice for the spherical wall radius is large enough to probe wavelengths much larger than the lattice spacing. The breaking of rotational invariance due to lattice regularization can be seen in the small splitting of the different $\mathrm{SO}(3, Z)$ representations comprising each orbital angular momentum multiplet. The elements of each $\mathrm{SO}(3, Z)$ representation however remain exactly degenerate.

In Fig. 3 we show the interacting two-particle energy spectrum for $S=0$ for the same wall radius. From the lattice data we find that the interaction is attractive in each of the $S=0$ channels. This is as one might expect since $V_{0}$ is negative definite. To illustrate the calculation of phase shifts, we take for example the $1^{1} S_{0}$ state. For the free particle system the $1^{1} S_{0}$ state has energy $0.9280 \mathrm{MeV}$, which corresponds with a continuum momentum of

$$
k_{\text {free }}=29.52 \mathrm{MeV} \text {. }
$$



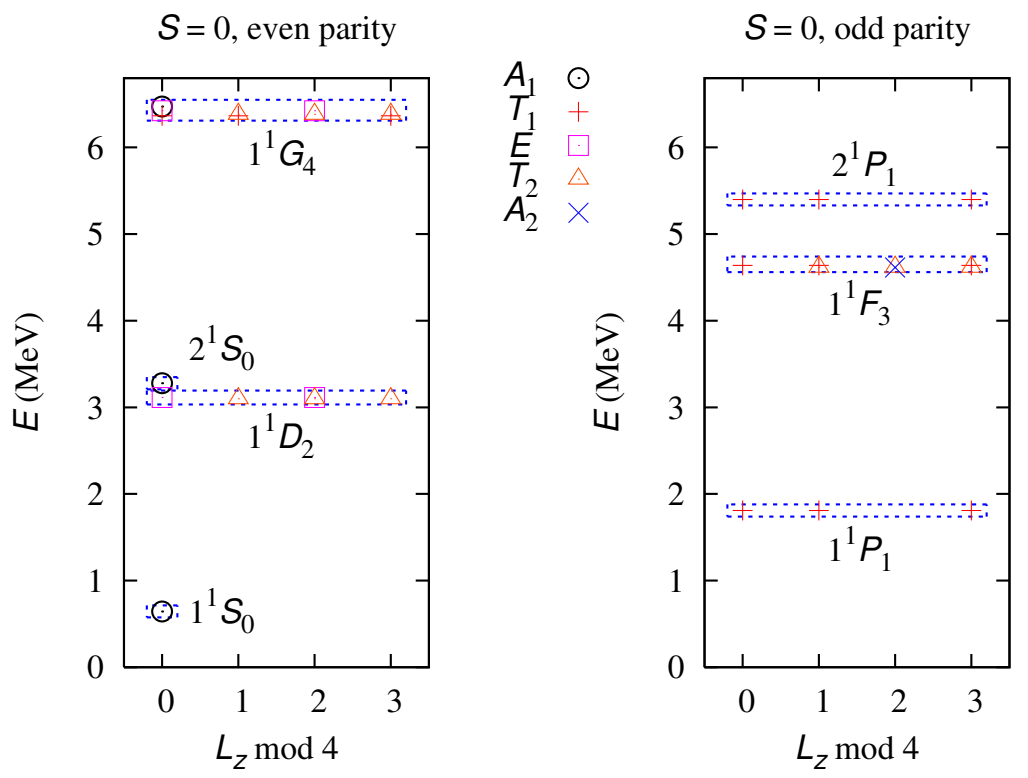

FIG. 3: Interacting spectrum for $S=0$ standing waves with $R_{\text {wall }}=10+\epsilon$ lattice units.

The $1^{1} S_{0}$ state has exactly one extremum and so the free wavefunction vanishes when

$$
\begin{gathered}
j_{0}\left(k_{\text {free }} R_{\text {wall }}\right)=0, \\
R_{\text {wall }}=\frac{\pi}{k_{\text {free }}}=0.1064 \mathrm{MeV}^{-1} .
\end{gathered}
$$

This corresponds with 10.64 lattice units, and is consistent with our wall starting at lattice distances greater than 10 lattice units. For the interacting system the $1^{1} S_{0}$ state has energy $0.6445 \mathrm{MeV}$. This corresponds with a continuum momentum of

$$
k=24.60 \mathrm{MeV} \text {. }
$$

Using (11) we find a ${ }^{1} S_{0}$ phase shift at $k=24.60 \mathrm{MeV}$ equal to

$$
\delta\left({ }^{1} S_{0}\right)=\tan ^{-1}\left[\frac{j_{0}\left(k R_{\text {wall }}\right)}{y_{0}\left(k R_{\text {wall }}\right)}\right]=30.0^{\circ} .
$$

We proceed in this manner for all of the $S=0$ phase shifts. In cases where the spin multiplet is slightly non-degenerate due to the lattice we use the average energy over all elements of the multiplet.

In Fig. 4 we compare lattice results and exact continuum results for the ${ }^{1} S_{0},{ }^{1} P_{1},{ }^{1} D_{2}$, ${ }^{1} F_{3}$, and ${ }^{1} G_{4}$ phase shifts as a function of the center-of-mass momentum $p_{\mathrm{CM}}$. For the lattice results we use $R_{\text {wall }}=10+\epsilon, 9+\epsilon$, and $8+\epsilon$ lattice units. In order of increasing momentum, 

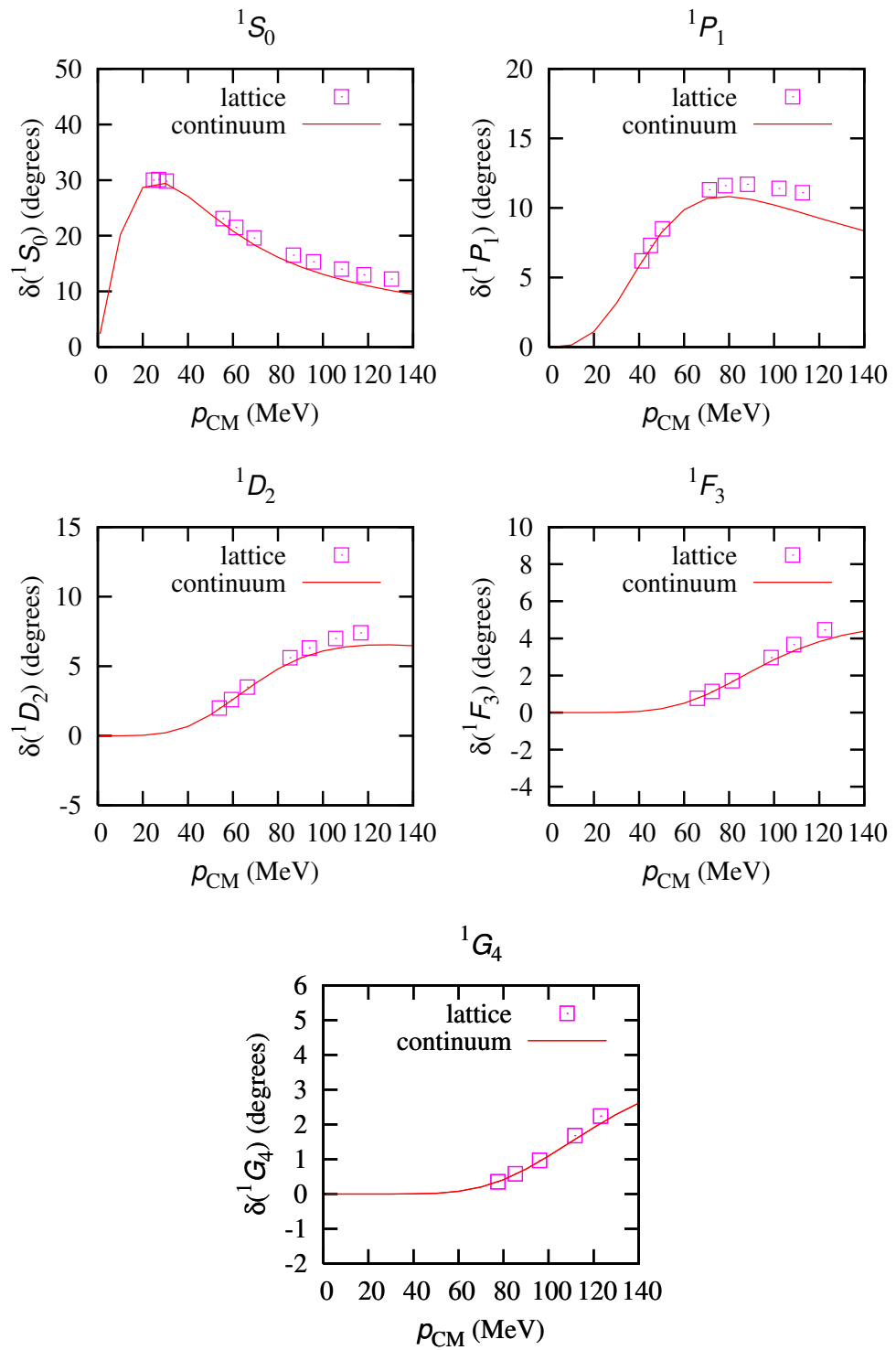

FIG. 4: $S=0$ phase shifts for $J \leq 4$. In order of increasing momentum the lattice data corresponds with the first radial excitation for $R_{\text {wall }}=10+\epsilon, 9+\epsilon$, and $8+\epsilon$; second radial excitation for $R_{\text {wall }}=10+\epsilon, 9+\epsilon$, and $8+\epsilon$; and so on.

the lattice data corresponds with the first radial excitation for $R_{\text {wall }}=10+\epsilon, 9+\epsilon$, and $8+\epsilon$; second radial excitation of $R_{\text {wall }}=10+\epsilon, 9+\epsilon$, and $8+\epsilon$; and so on. The continuum results are calculated by solving the Lippmann-Schwinger equation in momentum space.

The lattice results are within a few percent of exact results for momenta below $80 \mathrm{MeV}$. 
The error increases to $10 \%$ or $15 \%$ for momenta near $120 \mathrm{MeV}$. This is as good as can be expected without fine-tuning of the lattice action. The lattice spacing $a=(100 \mathrm{MeV})^{-1}$ corresponds with a momentum cutoff equal to $\pi / a=314 \mathrm{MeV}$. The quality of the results at higher spin is remarkable considering that $J_{z}$ can only be determined modulo 4 on the lattice using (11).

\section{LATTICE CALCULATION FOR $S=1$}

For intrinsic spin $S=1$ the tensor interaction in $V(\vec{r})$ must be included. We define the spin densities,

$$
\rho_{l}^{a^{\dagger}, a}(\vec{n})=\sum_{i, j=\uparrow, \downarrow} a_{i}^{\dagger}(\vec{n})\left[\sigma_{l}\right]_{i j} a_{j}(\vec{n}) \quad l=1,2,3 .
$$

The transfer matrix has the form

$$
\begin{aligned}
M \equiv: \exp [ & -H_{\text {free }} \alpha_{t}-\frac{\alpha_{t}}{2} \sum_{\vec{n}_{1}, \vec{n}_{2}} V_{0}\left(\vec{n}_{1}-\vec{n}_{2}\right) \rho^{a^{\dagger}, a}\left(\vec{n}_{1}\right) \rho^{a^{\dagger}, a}\left(\vec{n}_{2}\right) \\
& \left.-\frac{\alpha_{t}}{2 R_{0}^{2}} \sum_{\vec{n}_{1}, \vec{n}_{2}} \sum_{l, l^{\prime}=1,2,3} V_{0}\left(\vec{n}_{1}-\vec{n}_{2}\right) T_{l l^{\prime}}\left(\vec{n}_{1}-\vec{n}_{2}\right) \rho_{l}^{a^{\dagger}, a}\left(\vec{n}_{1}\right) \rho_{l^{\prime}}^{a^{\dagger}, a}\left(\vec{n}_{2}\right)\right]:,
\end{aligned}
$$

where

$$
T_{l l^{\prime}}(\vec{n})=3 n_{l} n_{l^{\prime}}-|\vec{n}|^{2} \delta_{l l^{\prime}}
$$

In Fig. 5 we show the interacting two-particle energy spectrum for $S=1$ for wall radius $R_{\text {wall }}=10+\epsilon$ lattice units.

The total angular momentum $J$ multiplets are deduced from the approximate degeneracy of $\mathrm{SO}(3, Z)$ representations comprising the multiplet decompositions in Table II. In some cases the accidental degeneracy of different $J$ multiplets makes this process difficult. For example the $1^{3} F(P)_{2}$ and $1^{3} F(H)_{4}$ multiplets are nearly degenerate for $R_{\text {wall }}=10+\epsilon$, as can be seen in Fig. 5. In such cases further information can be extracted by calculating the inner product of the standing wave with spherical harmonics $Y_{L, L_{z}}(\theta, \phi)$. In the infinite volume limit the $1^{3} S(D)_{1}$ bound state has an energy of $-0.170 \mathrm{MeV}$, in good agreement with the exact result $-0.155 \mathrm{MeV}$.

For $S=1$ we start with the uncoupled channels. Once the ${ }^{2 S+1} L_{J}$ multiplets are identified the process is exactly the same as for the $S=0$ case. In Fig. 6 we compare 

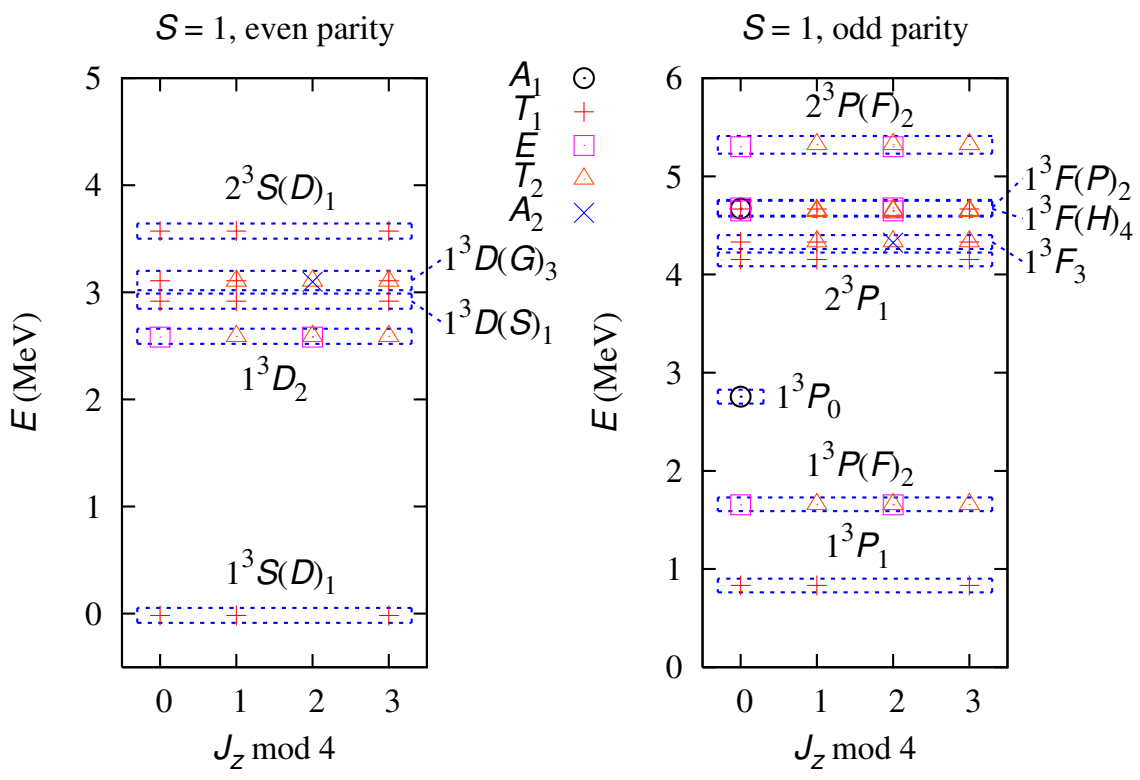

FIG. 5: Interacting spectrum for $S=1$ standing waves with $R_{\text {wall }}=10+\epsilon$ lattice units.

lattice results and exact continuum results for the ${ }^{3} P_{0},{ }^{3} P_{1},{ }^{3} D_{2},{ }^{3} F_{3}$, and ${ }^{3} G_{4}$ phase shifts. For the lattice results we again use $R_{\text {wall }}=10+\epsilon, 9+\epsilon$, and $8+\epsilon$ lattice units.

The tensor interaction produces a strong repulsion in the ${ }^{3} P_{0}$ channel, enough to overcome the attraction from the central force interaction. As in the $S=0$ case, the lattice results for the uncoupled $S=1$ channels are within a few percent of exact results for momenta below $80 \mathrm{MeV}$ and within $10 \%$ or $15 \%$ for momenta near $120 \mathrm{MeV}$.

\section{PARTIAL WAVE MIXING FOR $S=1$ AT ASYMPTOTICALLY LARGE RA- DIUS}

In the next few sections we consider partial-wave mixing between the partial waves $L=$ $J-1$ and $L=J+1$ using the Stapp parameterization [35]. It is convenient to use the two-component notation,

$$
\left[\begin{array}{c}
R_{J-1}(r) \\
R_{J+1}(r)
\end{array}\right],
$$



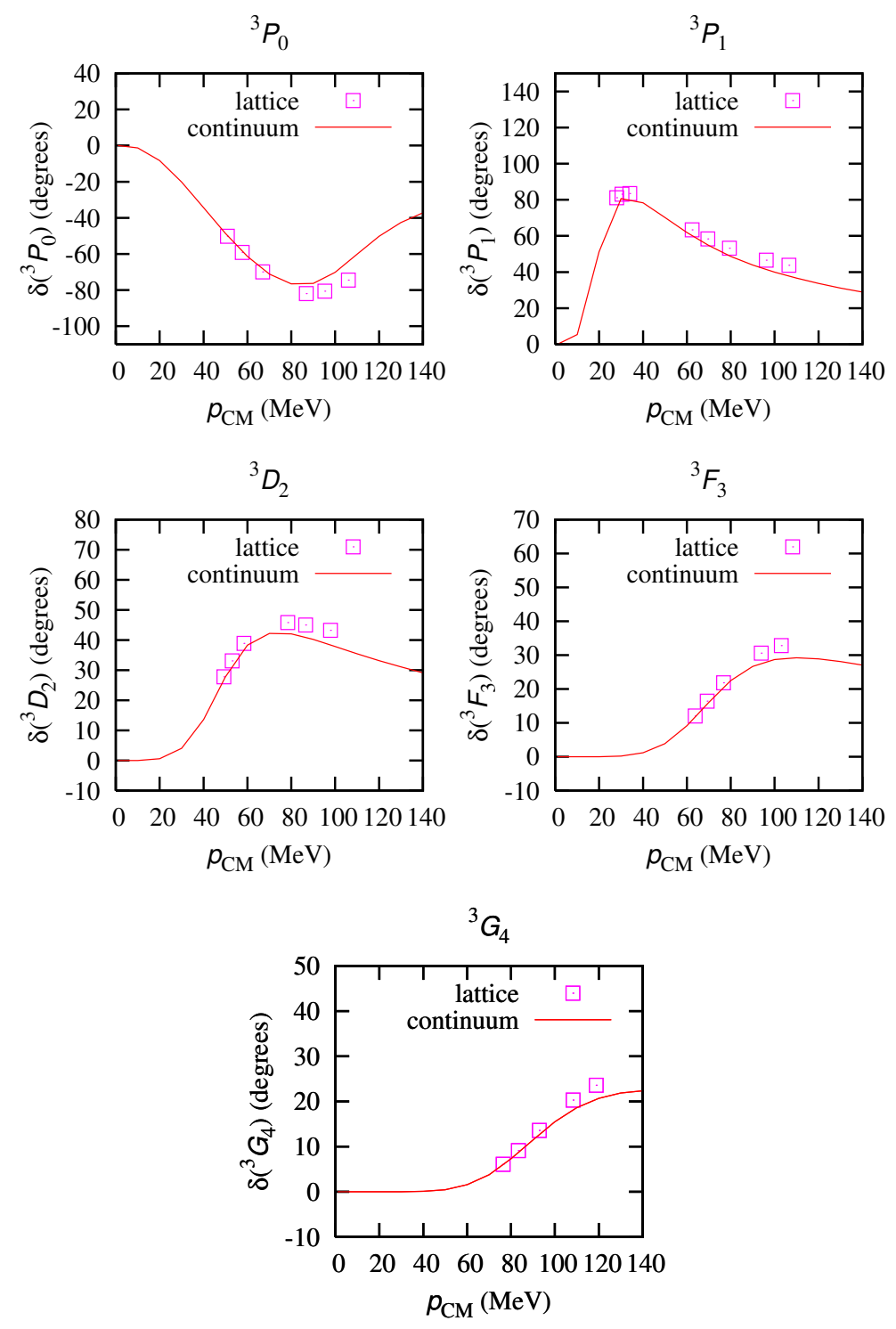

FIG. 6: Uncoupled $S=1$ phase shifts for $J \leq 4$. The lattice data corresponds with $R_{\text {wall }}=$ $10+\epsilon, 9+\epsilon$, and $8+\epsilon$.

for the radial part of the wavefunction in continuous space. The full expression is

$$
\begin{aligned}
& R_{J-1}(r) \sum_{L_{z}=-(J-1)}^{J-1} \sum_{S_{z}=-1}^{1} Y_{J-1, L_{z}}(\theta, \phi)\left\langle J-1, L_{z} ; 1, S_{z} \mid J, J_{z}\right\rangle \times\left|1, S_{z}\right\rangle \\
& +R_{J+1}(r) \sum_{L_{z}=-(J+1)}^{J+1} \sum_{S_{z}=-1}^{1} Y_{J+1, L_{z}}(\theta, \phi)\left\langle J+1, L_{z} ; 1, S_{z} \mid J, J_{z}\right\rangle \times\left|1, S_{z}\right\rangle,
\end{aligned}
$$


where $\left\langle L, L_{z} ; S, S_{z} \mid J, J_{z}\right\rangle$ denotes the usual Clebsch-Gordon coefficient for adding orbital angular momenta and intrinsic spin. Using this shorthand notation the S-matrix can be parametrized as a $2 \times 2$ matrix of the form

$$
\mathbf{S}=\left[\begin{array}{cc}
e^{i \delta_{J-1}} & 0 \\
0 & e^{i \delta_{J+1}}
\end{array}\right]\left[\begin{array}{cc}
\cos 2 \varepsilon_{J} & i \sin 2 \varepsilon_{J} \\
i \sin 2 \varepsilon_{J} & \cos 2 \varepsilon_{J}
\end{array}\right]\left[\begin{array}{cc}
e^{i \delta_{J-1}} & 0 \\
0 & e^{i \delta_{J+1}}
\end{array}\right]
$$

Let the incoming wave at asymptotically large radius $r$ be

$$
\Psi_{\text {in }}=-\frac{e^{-i(k r-J \pi / 2)}}{2 i k r}\left[\begin{array}{cc}
e^{-i \pi / 2} & 0 \\
0 & e^{i \pi / 2}
\end{array}\right]\left[\begin{array}{l}
C \\
D
\end{array}\right]
$$

Then the outgoing wave at large $r$ is [35, 36, 37]

$$
\begin{aligned}
\Psi_{\text {out }} & =\frac{e^{i(k r-J \pi / 2)}}{2 i k r}\left[\begin{array}{cc}
e^{i \pi / 2} & 0 \\
0 & e^{-i \pi / 2}
\end{array}\right] \mathbf{S}\left[\begin{array}{l}
C \\
D
\end{array}\right] \\
& =\frac{e^{i(k r-J \pi / 2)}}{2 i k r}\left[\begin{array}{c}
C e^{i \pi / 2} e^{2 i \delta_{J-1}} \cos 2 \varepsilon_{J}+i D e^{i \pi / 2} e^{i\left(\delta_{J-1}+\delta_{J+1}\right)} \sin 2 \varepsilon_{J} \\
D e^{-i \pi / 2} e^{2 i \delta_{J+1}} \cos 2 \varepsilon_{J}+i C e^{-i \pi / 2} e^{i\left(\delta_{J-1}+\delta_{J+1}\right)} \sin 2 \varepsilon_{J}
\end{array}\right] .
\end{aligned}
$$

It is convenient to define another unitary operator,

$$
\mathbf{W}=\left[\begin{array}{cc}
e^{-i \delta_{J-1}} & 0 \\
0 & e^{-i \delta_{J+1}}
\end{array}\right]\left[\begin{array}{cc}
\cos \varepsilon_{J} & -i \sin \varepsilon_{J} \\
-i \sin \varepsilon_{J} & \cos \varepsilon_{J}
\end{array}\right]
$$

so that

$$
\begin{aligned}
& \mathbf{W}^{\dagger}=\left[\begin{array}{cc}
\cos \varepsilon_{J} & i \sin \varepsilon_{J} \\
i \sin \varepsilon_{J} & \cos \varepsilon_{J}
\end{array}\right]\left[\begin{array}{cc}
e^{i \delta_{J-1}} & 0 \\
0 & e^{i \delta_{J+1}}
\end{array}\right], \\
& \mathbf{W}^{*}=\left[\begin{array}{cc}
e^{i \delta_{J-1}} & 0 \\
0 & e^{i \delta_{J+1}}
\end{array}\right]\left[\begin{array}{cc}
\cos \varepsilon_{J} & i \sin \varepsilon_{J} \\
i \sin \varepsilon_{J} & \cos \varepsilon_{J}
\end{array}\right] .
\end{aligned}
$$

These satisfy the identities

$$
\begin{gathered}
\mathbf{W} \mathbf{W}^{\dagger}=\mathbf{W}^{\dagger} \mathbf{W}=1, \\
\mathbf{W}^{*} \mathbf{W}^{\dagger}=\mathbf{S} .
\end{gathered}
$$

To construct a real-valued standing wave, we let the asymptotic incoming wave be

$$
-\frac{e^{-i(k r-J \pi / 2)}}{2 i k r}\left[\begin{array}{cc}
e^{-i \pi / 2} & 0 \\
0 & e^{i \pi / 2}
\end{array}\right] \mathbf{W}\left[\begin{array}{l}
C \\
D
\end{array}\right]
$$


for real numbers $C$ and $D$. Then the outgoing wave is

$$
\frac{e^{i(k r-J \pi / 2)}}{2 i k r}\left[\begin{array}{cc}
e^{i \pi / 2} & 0 \\
0 & e^{-i \pi / 2}
\end{array}\right] \mathbf{S W}\left[\begin{array}{c}
C \\
D
\end{array}\right]=\frac{e^{i(k r-J \pi / 2)}}{2 i k r}\left[\begin{array}{cc}
e^{i \pi / 2} & 0 \\
0 & e^{-i \pi / 2}
\end{array}\right] \mathbf{W}^{*}\left[\begin{array}{l}
C \\
D
\end{array}\right] .
$$

The resulting standing wave is then

$$
\begin{aligned}
\Psi & =2 \operatorname{Re}\left\{\frac{e^{i(k r-J \pi / 2)}}{2 i k r}\left[\begin{array}{cc}
e^{i \pi / 2} & 0 \\
0 & e^{-i \pi / 2}
\end{array}\right] \mathbf{W}^{*}\left[\begin{array}{l}
C \\
D
\end{array}\right]\right\} \\
& =\frac{1}{k r}\left[\begin{array}{l}
C \sin \left(k r-\frac{J-1}{2} \pi+\delta_{J-1}\right) \cos \varepsilon_{J}+D \cos \left(k r-\frac{J-1}{2} \pi+\delta_{J-1}\right) \sin \varepsilon_{J} \\
D \sin \left(k r-\frac{J+1}{2} \pi+\delta_{J+1}\right) \cos \varepsilon_{J}+C \cos \left(k r-\frac{J+1}{2} \pi+\delta_{J+1}\right) \sin \varepsilon_{J}
\end{array}\right] .
\end{aligned}
$$

We now impose a hard spherical wall boundary at $r=R_{\text {wall }}$. Both partial waves vanish at the wall, and we define the angle $\Delta$ so that

$$
-\Delta=k R_{\text {wall }}-\frac{J-1}{2} \pi .
$$

Then

$$
\frac{C}{D} \tan \left(-\Delta+\delta_{J-1}\right)=-\tan \varepsilon_{J}
$$

and

$$
\frac{D}{C} \tan \left(-\Delta-\pi+\delta_{J+1}\right)=\frac{D}{C} \tan \left(-\Delta+\delta_{J+1}\right)=-\tan \varepsilon_{J}
$$

In general there are two solutions for $\Delta$ per angular interval $\pi$. In the asymptotic case where $R_{\text {wall }} \gg k^{-1}$ the spacing between energy levels becomes infinitesimal. We can therefore choose two independent solutions $\Delta^{I}$ and $\Delta^{I I}$ with nearly the same values of $k$, and we neglect the difference in $k$ in the following steps.

The solutions $\Delta^{I}$ and $\Delta^{I I}$ satisfy

$$
\tan \left(-\Delta^{I, I I}+\delta_{J-1}\right) \tan \left(-\Delta^{I, I I}+\delta_{J+1}\right)=\tan ^{2} \varepsilon_{J}
$$

The standing wave now looks like

$$
\begin{aligned}
\Psi & =\frac{1}{k r}\left[\begin{array}{c}
C \sin \left(k r-\frac{J-1}{2} \pi+\delta_{J-1}\right) \cos \varepsilon_{J}-C \frac{\sin \left(-\Delta+\delta_{J-1}\right)}{\cos \left(-\Delta+\delta_{J-1}\right)} \cos \left(k r-\frac{J-1}{2} \pi+\delta_{J-1}\right) \cos \varepsilon_{J} \\
-C \sin \left(k r-\frac{J+1}{2} \pi+\delta_{J+1}\right) \frac{\cos \left(-\Delta+\delta_{J+1}\right)}{\sin \left(-\Delta+\delta_{J+1}\right)} \sin \varepsilon_{J}+C \cos \left(k r-\frac{J+1}{2} \pi+\delta_{J+1}\right) \sin \varepsilon_{J}
\end{array}\right] \\
= & \frac{C}{k r}\left[\begin{array}{c}
\frac{\cos \varepsilon_{J}}{\cos \left(-\Delta+\delta_{J-1}\right)} \sin \left[k r-\frac{J-1}{2} \pi+\Delta\right] \\
-\frac{\sin \varepsilon_{J}}{\sin \left(-\Delta+\delta_{J+1}\right)} \sin \left[k r-\frac{J+1}{2} \pi+\Delta\right]
\end{array}\right] .
\end{aligned}
$$


There are two linearly independent real-valued standing wave solutions. We write them as

$$
\Psi^{I, I I} \propto \frac{1}{k r}\left[\begin{array}{c}
A_{J-1}^{I, I I} \sin \left(k r-\frac{J-1}{2} \pi+\Delta^{I, I I}\right) \\
A_{J+1}^{I, I I} \sin \left(k r-\frac{J+1}{2} \pi+\Delta^{I, I I}\right)
\end{array}\right] .
$$

Then

$$
A_{J-1}^{I, I I} \tan \varepsilon_{J}=-A_{J+1}^{I, I I} \frac{\sin \left(-\Delta^{I, I I}+\delta_{J+1}\right)}{\cos \left(-\Delta^{I, I I}+\delta_{J-1}\right)} .
$$

Only three of the constraints in (42) and (45) are needed to determine the parameters $\delta_{J-1}, \delta_{J+1}$, and $\varepsilon_{J}$. Therefore one constraint, for example

$$
A_{J-1}^{I I} \tan \varepsilon_{J}=-A_{J+1}^{I I} \frac{\sin \left(-\Delta^{I I}+\delta_{J+1}\right)}{\cos \left(-\Delta^{I I}+\delta_{J-1}\right)},
$$

must be redundant. This makes sense in light of the symmetry associated with the interchange of the two solutions $\Delta^{I}$ and $\Delta^{I I}$. The orthogonality of $\Psi^{I}$ and $\Psi^{I I}$ implies

$$
\begin{gathered}
A_{J-1}^{I} A_{J-1}^{I I}+A_{J+1}^{I} A_{J+1}^{I I}=0, \\
\frac{A_{J+1}^{I}}{A_{J-1}^{I}}=-\frac{A_{J-1}^{I I}}{A_{J+1}^{I I}} .
\end{gathered}
$$

From this one can derive (46) from (42) and (45).

\section{EXPANSION FOR SMALL $\varepsilon_{J}$}

For our test potential we have chosen the tensor force to be very strong, and so the mixing angles are quite large. In several real physical systems however, for example nucleon-nucleon scattering at low momenta, the mixing angles are quite small. For small $\varepsilon_{J}$ it is useful to

expand around $\varepsilon_{J}=0$. We choose $\Delta^{I}$ to be the solution that equals $\delta_{J-1}$ at zero mixing, and $\Delta^{I I}$ to be the solution that equals $\delta_{J+1}$ at zero mixing. Since

$$
\tan \left(-\Delta^{I}+\delta_{J-1}\right) \tan \left(-\Delta^{I}+\delta_{J+1}\right)=\tan ^{2} \varepsilon_{J}
$$

the first correction to $\Delta^{I}$ and $\Delta^{I I}$ comes at order $O\left(\varepsilon_{J}^{2}\right)$,

$$
\begin{gathered}
\Delta^{I}=\delta_{J-1}+c^{I} \varepsilon_{J}^{2}+O\left(\varepsilon_{J}^{4}\right), \\
\Delta^{I I}=\delta_{J+1}+c^{I I} \varepsilon_{J}^{2}+O\left(\varepsilon_{J}^{4}\right) .
\end{gathered}
$$

We find

$$
-c^{I} \varepsilon_{J}^{2} \tan \left(\delta_{J+1}-\delta_{J-1}\right)=\varepsilon_{J}^{2}+O\left(\varepsilon_{J}^{4}\right)
$$




$$
c^{I}=-\frac{1}{\tan \left(\delta_{J+1}-\delta_{J-1}\right)}
$$

Similarly

$$
\begin{gathered}
-c^{I I} \varepsilon_{J}^{2} \tan \left(\delta_{J-1}-\delta_{J+1}\right)=\varepsilon_{J}^{2}+O\left(\varepsilon_{J}^{4}\right), \\
c^{I I}=\frac{1}{\tan \left(\delta_{J+1}-\delta_{J-1}\right)} .
\end{gathered}
$$

Since

$$
A_{J-1}^{I} \tan \varepsilon_{J}=-A_{J+1}^{I} \frac{\sin \left(-\Delta^{I}+\delta_{J+1}\right)}{\cos \left(-\Delta^{I}+\delta_{J-1}\right)}
$$

we have

$$
\sin \left(\delta_{J+1}-\delta_{J-1}\right)=-\frac{A_{J-1}^{I} \varepsilon_{J}}{A_{J+1}^{I}}+O\left(\varepsilon_{J}^{2}\right) .
$$

Up to $O\left(\varepsilon_{J}^{2}\right)$ then

$$
\sin \left(\Delta^{I I}-\Delta^{I}\right)=-\frac{A_{J-1}^{I} \varepsilon_{J}}{A_{J+1}^{I}}+O\left(\varepsilon_{J}^{2}\right),
$$

or

$$
\varepsilon_{J}=-\frac{A_{J+1}^{I}}{A_{J-1}^{I}} \sin \left(\Delta^{I I}-\Delta^{I}\right)+O\left(\varepsilon_{J}^{3}\right) .
$$

Similarly we have

$$
A_{J-1}^{I I} \tan \varepsilon_{J}=-A_{J+1}^{I I} \frac{\sin \left(-\Delta^{I I}+\delta_{J+1}\right)}{\cos \left(-\Delta^{I I}+\delta_{J-1}\right)}
$$

and so

$$
\begin{aligned}
A_{J-1}^{I I} & =-A_{J+1}^{I I} \frac{-c^{I I} \varepsilon_{J}^{2}}{\varepsilon_{J} \cos \left(-\delta_{J+1}+\delta_{J-1}\right)}+O\left(\varepsilon_{J}^{3}\right) \\
& =A_{J+1}^{I I} \frac{\varepsilon_{J}}{\sin \left(\delta_{J+1}-\delta_{J-1}\right)}+O\left(\varepsilon_{J}^{3}\right),
\end{aligned}
$$

or

$$
\sin \left(\delta_{J+1}-\delta_{J-1}\right)=\frac{A_{J+1}^{I I} \varepsilon_{J}}{A_{J-1}^{I I}}+O\left(\varepsilon_{J}^{2}\right) .
$$

Up to order $O\left(\varepsilon_{J}^{2}\right)$ we find

$$
\varepsilon_{J}=\frac{A_{J-1}^{I I}}{A_{J+1}^{I I}} \sin \left(\Delta^{I I}-\Delta^{I}\right)+O\left(\varepsilon_{J}^{3}\right) .
$$

This is consistent with (59) given the orthogonality condition,

$$
\frac{A_{J+1}^{I}}{A_{J-1}^{I}}=-\frac{A_{J-1}^{I I}}{A_{J+1}^{I I}} .
$$




\section{HARD SPHERICAL WALL AT NON-ASYMPTOTIC RADIUS}

The constraints in (42) and (45) hold if the spherical wall is at asymptotically large radius, $k R_{\text {wall }} \gg 1$. In this limit the nodes of the spherical Bessel functions $j_{J-1}(k r)$ and $j_{J+1}(k r)$ coincide, and we determine one shift angle for both partial waves,

$$
-\Delta=k R_{\text {wall }}-\frac{J-1}{2} \pi
$$

However for numerical calculations it is more convenient to work with smaller values of $k R_{\text {wall. }}$ In this case the coincidence of the nodes for $L=J-1$ and $L=J+1$ at the wall boundary does not automatically imply the coincidence of nodes for $k r \gg 1$. Nevertheless we can still work with the asymptotic form for $k r \gg 1$ if the node remains at $R_{\text {wall }}$ but the spherical wall is removed,

$$
\Psi=\frac{1}{k r}\left[\begin{array}{c}
C \sin \left(k r-\frac{J-1}{2} \pi+\delta_{J-1}\right) \cos \varepsilon_{J}+D \cos \left(k r-\frac{J-1}{2} \pi+\delta_{J-1}\right) \sin \varepsilon_{J} \\
D \sin \left(k r-\frac{J+1}{2} \pi+\delta_{J+1}\right) \cos \varepsilon_{J}+C \cos \left(k r-\frac{J+1}{2} \pi+\delta_{J+1}\right) \sin \varepsilon_{J}
\end{array}\right] .
$$

Instead of one angle $\Delta$, we define two angles $\Delta_{J-1}$ and $\Delta_{J+1}$ so that the $J-1$ partial wave vanishes when

$$
-\Delta_{J-1}=k r-\frac{J-1}{2} \pi,
$$

and the $J+1$ partial wave vanishes when

$$
-\Delta_{J+1}=k r-\frac{J+1}{2} \pi
$$

Then

$$
\frac{C}{D} \tan \left(-\Delta_{J-1}+\delta_{J-1}\right)=-\tan \varepsilon_{J}
$$

and

$$
\frac{D}{C} \tan \left(-\Delta_{J+1}+\delta_{J+1}\right)=-\tan \varepsilon_{J} .
$$

In general for a given $k$, there are two pairs of solutions $\left(\Delta_{J-1}, \Delta_{J+1}\right)$ per angular interval $\pi$ corresponding with different positions of the nodes. However instead of taking $k$ as the independent variable, we consider the location of the spherical wall radius as the independent variable. We denote the two solutions as $\left(\Delta_{J-1}^{I}, \Delta_{J+1}^{I}, k^{I}\right)$ and $\left(\Delta_{J-1}^{I I}, \Delta_{J+1}^{I I}, k^{I I}\right)$. We have

$$
\tan \left(-\Delta_{J-1}^{I}+\delta_{J-1}\right) \tan \left(-\Delta_{J+1}^{I}+\delta_{J+1}\right)=\tan ^{2} \varepsilon_{J}
$$


at momentum $k^{I}$, and

$$
\tan \left(-\Delta_{J-1}^{I I}+\delta_{J-1}\right) \tan \left(-\Delta_{J+1}^{I I}+\delta_{J+1}\right)=\tan ^{2} \varepsilon_{J}
$$

at momentum $k^{I I}$.

The standing wave now looks like

$$
\begin{aligned}
\Psi & =\frac{1}{k r}\left[\begin{array}{c}
C \sin \left(k r-\frac{J-1}{2} \pi+\delta_{J-1}\right) \cos \varepsilon_{J}+D \cos \left(k r-\frac{J-1}{2} \pi+\delta_{J-1}\right) \sin \varepsilon_{J} \\
D \sin \left(k r-\frac{J+1}{2} \pi+\delta_{J+1}\right) \cos \varepsilon_{J}+C \cos \left(k r-\frac{J+1}{2} \pi+\delta_{J+1}\right) \sin \varepsilon_{J}
\end{array}\right] \\
& =\frac{C}{k r}\left[\begin{array}{c}
\frac{\cos \varepsilon_{J}}{\cos \left(-\Delta_{J-1}+\delta_{J-1}\right)} \sin \left[k r-\frac{J-1}{2} \pi+\Delta_{J-1}\right] \\
-\frac{\sin \varepsilon_{J}}{\sin \left(-\Delta_{J+1}+\delta_{J+1}\right)} \sin \left[k r-\frac{J+1}{2} \pi+\Delta_{J+1}\right]
\end{array}\right] .
\end{aligned}
$$

We write these as

$$
\Psi^{I} \propto \frac{1}{k^{I} r}\left[\begin{array}{c}
A_{J-1}^{I} \sin \left(k^{I} r-\frac{J-1}{2} \pi+\Delta_{J-1}^{I}\right) \\
A_{J+1}^{I} \sin \left(k^{I} r-\frac{J+1}{2} \pi+\Delta_{J+1}^{I}\right)
\end{array}\right]
$$

and

$$
\Psi^{I I} \propto \frac{1}{k^{I I} r}\left[\begin{array}{c}
A_{J-1}^{I I} \sin \left(k^{I I} r-\frac{J-1}{2} \pi+\Delta_{J-1}^{I I}\right) \\
A_{J+1}^{I I} \sin \left(k^{I I} r-\frac{J+1}{2} \pi+\Delta_{J+1}^{I I}\right)
\end{array}\right] .
$$

So the generalization of equations (42) and (45) to the non-asymptotic case is

$$
\begin{gathered}
\tan \left(-\Delta_{J-1}^{I}+\delta_{J-1}\right) \tan \left(-\Delta_{J+1}^{I}+\delta_{J+1}\right)=\tan ^{2} \varepsilon_{J}, \\
\tan \left(-\Delta_{J-1}^{I I}+\delta_{J-1}\right) \tan \left(-\Delta_{J+1}^{I I}+\delta_{J+1}\right)=\tan ^{2} \varepsilon_{J}, \\
A_{J-1}^{I} \tan \varepsilon_{J}=-A_{J+1}^{I} \frac{\sin \left(-\Delta_{J+1}^{I}+\delta_{J+1}\right)}{\cos \left(-\Delta_{J-1}^{I}+\delta_{J-1}\right)}, \\
A_{J-1}^{I I} \tan \varepsilon_{J}=-A_{J+1}^{I I} \frac{\sin \left(-\Delta_{J+1}^{I I}+\delta_{J+1}\right)}{\cos \left(-\Delta_{J-1}^{I I}+\delta_{J-1}\right)} .
\end{gathered}
$$

We note that the phase shifts and mixing angle in (176) and (178) are at momentum $k^{I}$ while the phase shifts and mixing angle in (77) and (79) are at momentum $k^{I I}$. In the calculation one must in general interpolate between values for $k=k^{I}$ and $k=k^{I I}$. However the need for interpolation is largely eliminated by considering only close pairs of values $k^{I} \approx k^{I I}$ in solving (76)-(79). This can be done for example by considering the $(n+1)^{\text {st }}$-radial excitation of $L=J-1$ together with the $n^{\text {th }}$-radial excitation of $L=J+1$. In this scheme we use

$$
\begin{aligned}
& \tan \left(-\Delta_{J-1}^{I}+\delta_{J-1}\left(k^{I}\right)\right) \tan \left(-\Delta_{J+1}^{I}+\delta_{J+1}\left(k^{I}\right)\right)=\tan ^{2}\left[\varepsilon_{J}\left(k^{I}\right)\right], \\
& \tan \left(-\Delta_{J-1}^{I I}+\delta_{J-1}\left(k^{I}\right)\right) \tan \left(-\Delta_{J+1}^{I I}+\delta_{J+1}\left(k^{I}\right)\right) \approx \tan ^{2}\left[\varepsilon_{J}\left(k^{I}\right)\right],
\end{aligned}
$$




$$
A_{J-1}^{I} \tan \left[\varepsilon_{J}\left(k^{I}\right)\right]=-A_{J+1}^{I} \frac{\sin \left(-\Delta_{J+1}^{I}+\delta_{J+1}\left(k^{I}\right)\right)}{\cos \left(-\Delta_{J-1}^{I}+\delta_{J-1}\left(k^{I}\right)\right)},
$$

for the phase shifts and mixing angle at $k=k^{I}$, and

$$
\begin{gathered}
\tan \left(-\Delta_{J-1}^{I}+\delta_{J-1}\left(k^{I I}\right)\right) \tan \left(-\Delta_{J+1}^{I}+\delta_{J+1}\left(k^{I I}\right)\right) \approx \tan ^{2}\left[\varepsilon_{J}\left(k^{I I}\right)\right], \\
\tan \left(-\Delta_{J-1}^{I I}+\delta_{J-1}\left(k^{I I}\right)\right) \tan \left(-\Delta_{J+1}^{I I}+\delta_{J+1}\left(k^{I I}\right)\right)=\tan ^{2}\left[\varepsilon_{J}\left(k^{I I}\right)\right], \\
A_{J-1}^{I I} \tan \left[\varepsilon_{J}\left(k^{I I}\right)\right]=-A_{J+1}^{I I} \frac{\sin \left(-\Delta_{J+1}^{I I}+\delta_{J+1}\left(k^{I I}\right)\right)}{\cos \left(-\Delta_{J-1}^{I I}+\delta_{J-1}\left(k^{I I}\right)\right)}
\end{gathered}
$$

for the phase shifts and mixing angle at $k=k^{I I}$. This is the technique we use for the lattice results presented here. If more accuracy is required then this pair technique for $k^{I} \approx k^{I I}$ can be used as a starting point to determine numerical derivatives for the phase shifts and mixing angle. Then the equations (76) $-(79)$ can be solved again while keeping all terms at order $O\left(k^{I}-k^{I I}\right)$.

In the limit of small mixing the expressions in (80)-(85) reduce to

$$
\begin{gathered}
\delta_{J-1}\left(k^{I}\right)=\Delta_{J-1}^{I}+\frac{\varepsilon_{J}^{2}\left(k^{I}\right)}{\tan \left(-\Delta_{J+1}^{I}+\delta_{J+1}\left(k^{I}\right)\right)}+O\left(\varepsilon_{J}^{4}\right), \\
\varepsilon_{J}\left(k^{I}\right)=-\frac{A_{J+1}^{I}}{A_{J-1}^{I}} \sin \left(\Delta_{J+1}^{I I}-\Delta_{J+1}^{I}\right)+O\left(\varepsilon_{J}^{3}\right),
\end{gathered}
$$

at $k=k^{I}$ and

$$
\begin{gathered}
\delta_{J+1}\left(k^{I I}\right)=\Delta_{J+1}^{I I}+\frac{\varepsilon_{J}^{2}\left(k^{I I}\right)}{\tan \left(-\Delta_{J-1}^{I I}+\delta_{J-1}\left(k^{I I}\right)\right)}+O\left(\varepsilon_{J}^{4}\right), \\
\varepsilon_{J}\left(k^{I I}\right)=\frac{A_{J-1}^{I I}}{A_{J+1}^{I I}} \sin \left(\Delta_{J-1}^{I I}-\Delta_{J-1}^{I}\right)+O\left(\varepsilon_{J}^{3}\right) .
\end{gathered}
$$

at $k=k^{I I}$.

\section{RESULTS FOR $S=1$ COUPLED CHANNELS}

In Fig. 7 we show lattice and continuum results for ${ }^{3} S_{1},{ }^{3} D_{1}$ partial waves and $J=1$ mixing angle $\varepsilon_{1}$. The ${ }^{3} P_{2},{ }^{3} F_{2}$ partial waves and mixing angle $\varepsilon_{2}$ are shown in Fig. 8. The ${ }^{3} D_{3},{ }^{3} G_{3}$ partial waves and mixing angle $\varepsilon_{3}$ are shown in Fig. 9. The ${ }^{3} F_{4},{ }^{3} H_{4}$ partial waves and mixing angle $\varepsilon_{4}$ are shown in Fig. 10. As before we use $R_{\text {wall }}=10+\epsilon, 9+\epsilon$, and $8+\epsilon$ 

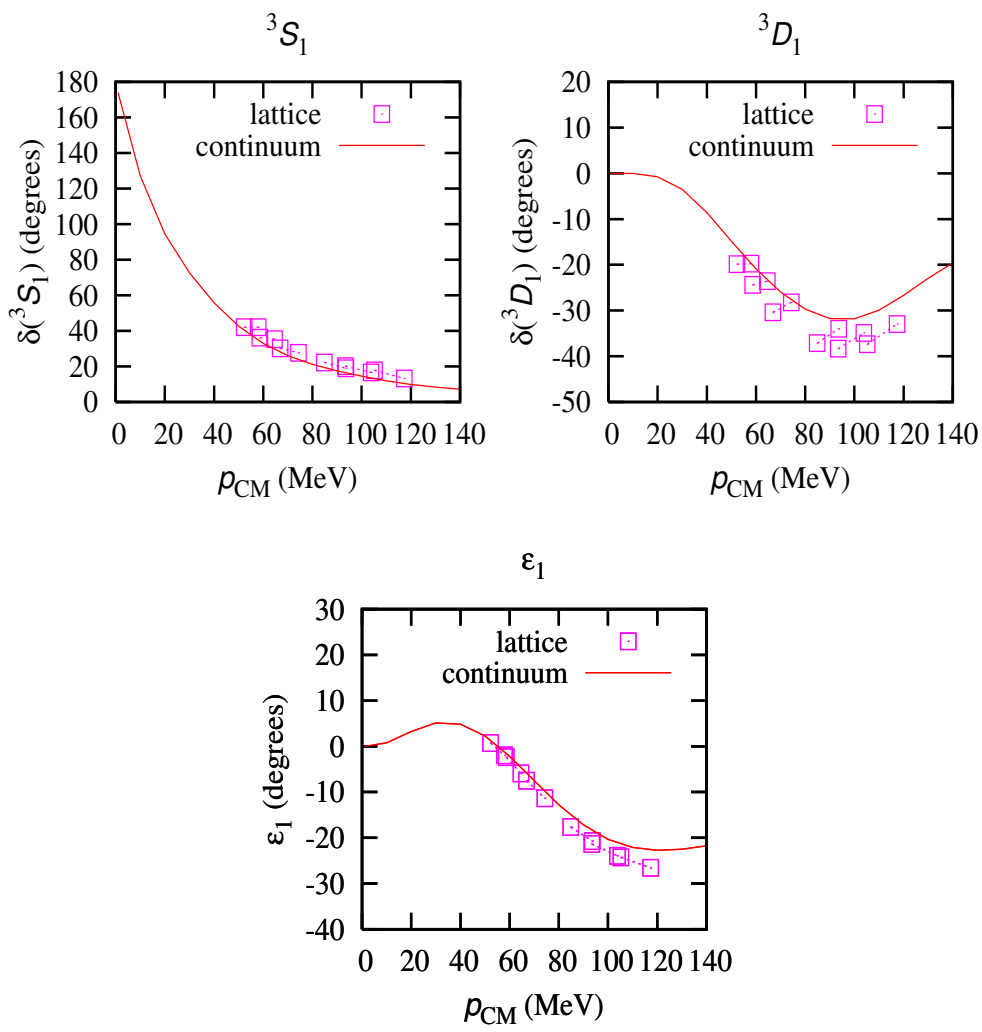

FIG. 7: Coupled partial waves and mixing angle for $J=1$. The pairs of points connected by dotted lines indicate pairs of solutions at $k=k^{I}$ and $k=k^{I I}$.

lattice units. The pairs of points connected by dotted lines indicate pairs of solutions at $k=k^{I}$ and $k=k^{I I}$. The partial-wave ratios

$$
\frac{A_{J-1}^{I}}{A_{J+1}^{I}}, \frac{A_{J-1}^{I I}}{A_{J+1}^{I I}},
$$

are determined by computing the inner product of the standing wave near the spherical wall with spherical harmonics.

The lattice results in the coupled channels are somewhat less accurate than the $S=0$ results and uncoupled $S=1$ results. However they are still within $20 \%$ of the exact results up to momentum $120 \mathrm{MeV}$. The leading error appears to come from mixing with extraneous channels due to broken rotational invariance on the lattice. For example the ${ }^{3} S_{1^{-}}{ }^{3} D_{1}$ standing waves have a small but non-negligible amount of a ${ }^{3} D_{3}$ component. Nevertheless overall the lattice results are remarkably good for partial-wave mixing at higher total angular 

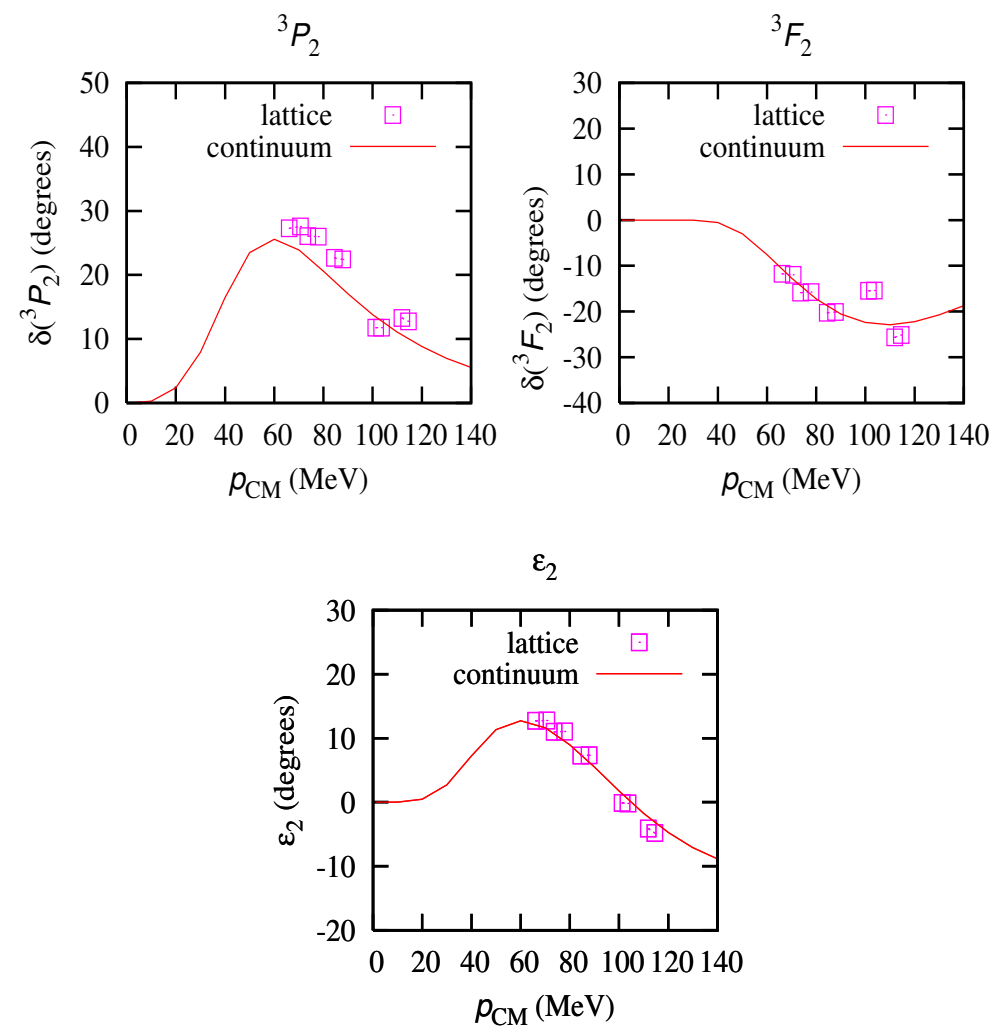

FIG. 8: Coupled partial waves and mixing angle for $J=2$.

momentum.

\section{SUMMARY AND DISCUSSION}

We have discussed a general technique for measuring phase shifts and mixing angles for two-particle scattering on the lattice. In the center-of-mass frame we impose a hard spherical wall at large fixed radius $R_{\text {wall }}$. For channels without mixing we identify total angular momentum $J$ multiplets by the approximate degeneracy of $\mathrm{SO}(3, Z)$ representations and calculate phase shifts from the energies of the spherical standing waves. For channels with partial-wave mixing, further information is extracted by decomposing the standing wave at the wall boundary into spherical harmonics. The coupled-channels equations are then solved to extract the phase shifts and mixing angles. The method was tested by computing phase shifts and mixing angles for $J$ less than or equal to 4 for an attractive Gaussian potential 

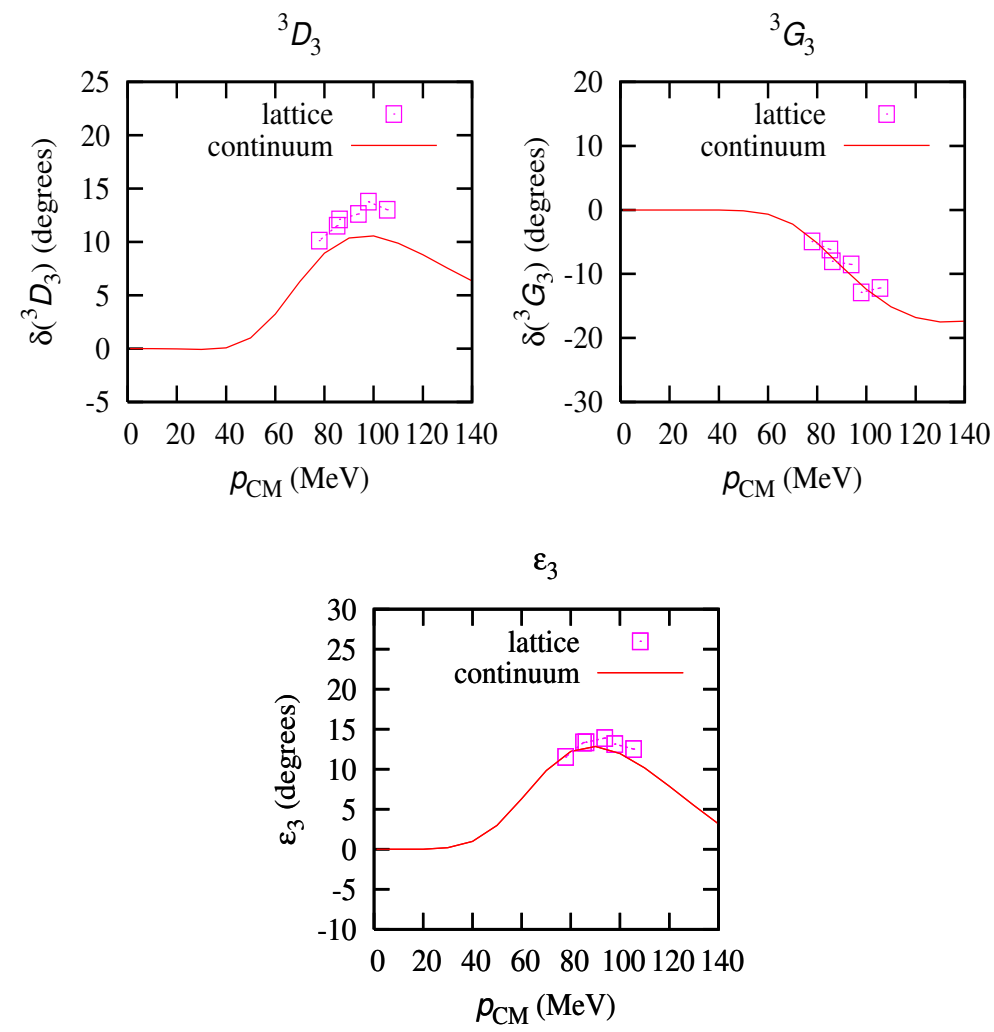

FIG. 9: Coupled partial waves and mixing angle for $J=3$.

with both central and tensor force parts. The Gaussian envelope had a characteristic size of $R_{0}=2 \times 10^{-2} \mathrm{MeV}^{-1}$, and the strength was tuned to produce a shallow ${ }^{3} S(D)_{1}$ bound state. At spatial lattice spacing $a=(100 \mathrm{MeV})^{-1}$ and temporal lattice spacing $a_{t}=(70$ $\mathrm{MeV})^{-1}$ we found agreement with the exact results at the 10\%-20\% level for momenta up to $120 \mathrm{MeV}$.

The hard spherical wall removes extra copies of the two-particle interactions due to the periodic boundaries of the lattice. This appears to be important for measuring phase shifts and mixing angles at higher energies and higher angular momentum. For comparison we show in Fig. 11 the same test potential spectrum for intrinsic spin $S=1$ on a $12 \times 12 \times 12$ lattice with periodic boundaries. If we compare with Fig. 5 we see that the $\mathrm{SO}(3, Z)$ representations in the various total angular momentum $J$ multiplets have been split apart much further. It is easy to identify the lowest-lying standing waves but difficult to clearly discern much beyond this. 

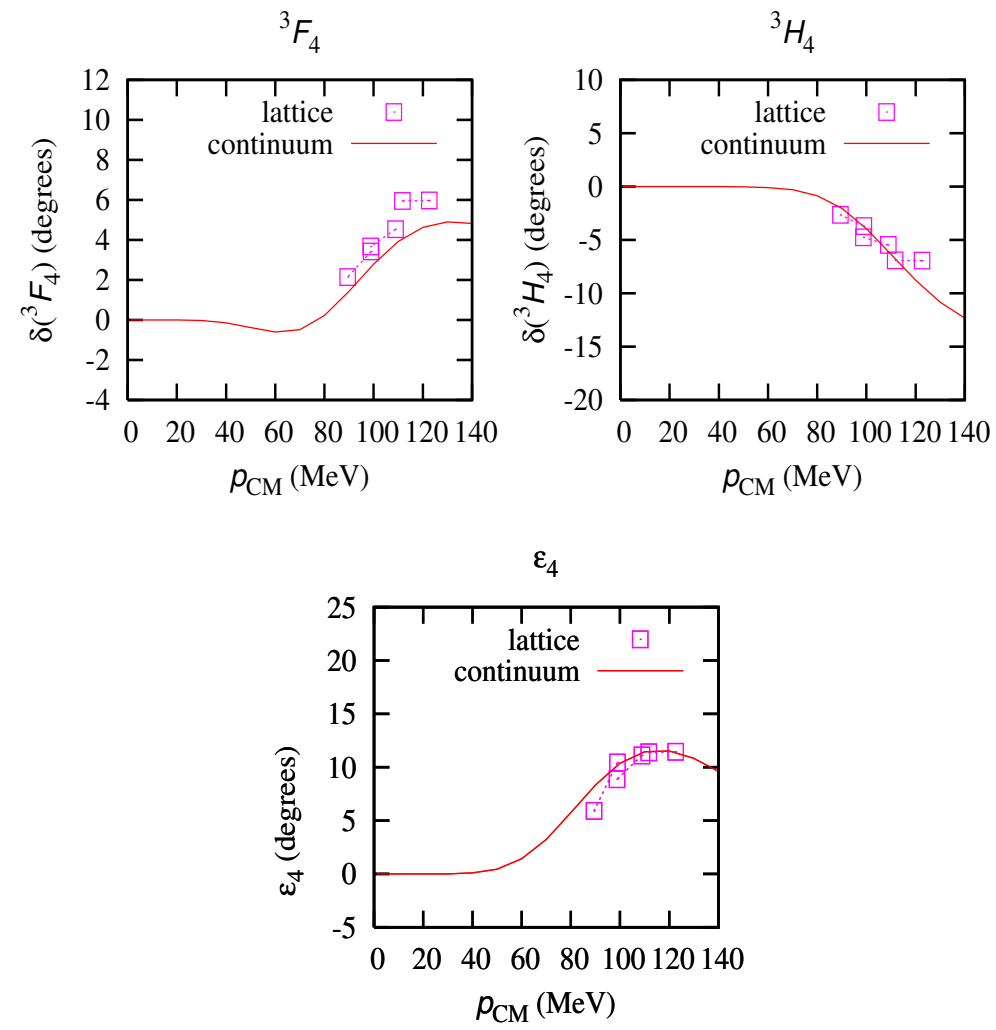

FIG. 10: Coupled partial waves and mixing angle for $J=4$.
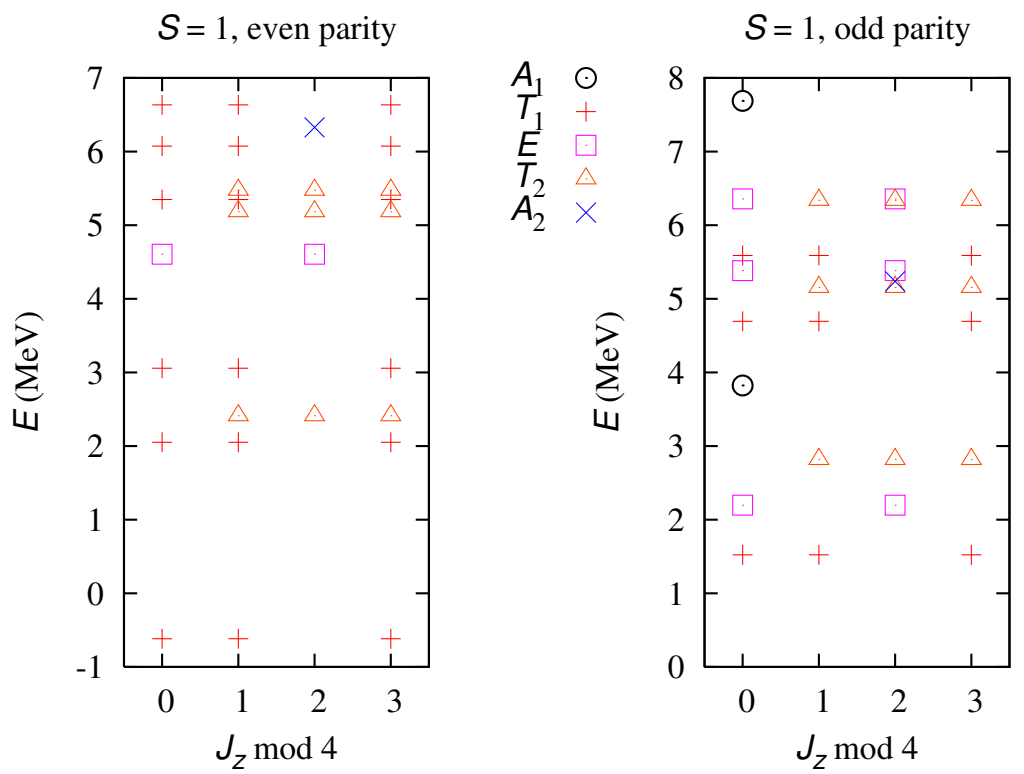

FIG. 11: Interacting spectrum for $S=1$ standing waves for a $12 \times 12 \times 12$ periodic lattice. 
The method we have presented can be applied directly to any nonrelativistic effective theory of point particles on the lattice. There is a long list of interesting few- and manybody systems one could consider on the lattice. For example one interesting system is that of atoms and molecules with long-range dipole interactions,

$$
V_{\text {dipole-dipole }}(\vec{r}) \propto-\frac{1}{4 \pi r^{3}}\left[3\left(\hat{r} \cdot \vec{\mu}_{1}\right)\left(\hat{r} \cdot \vec{\mu}_{2}\right)-\vec{\mu}_{1} \cdot \vec{\mu}_{2}\right] .
$$

There is interest both in magnetic dipole interactions of cold atoms [38, 39, 40, 41, 42] and electric dipole interactions in cold polar molecules [43, 44, 45, 46]. These spin-changing interactions are important for determining topological structures of the many-body ground state as well as practical issues impacting evaporative cooling in magnetic traps.

We will discuss in some detail the application to low-energy nuclear physics in a forthcoming paper. One important low-energy interaction between nucleons is the exchange of a virtual pion, producing spin-dependent forces of the form

$$
\begin{aligned}
V_{1 \pi}(\vec{r}) & =\left(\frac{g_{A}}{2 f_{\pi}}\right)^{2}\left(\boldsymbol{\tau}_{1} \cdot \boldsymbol{\tau}_{2}\right) \\
& \times\left\{\frac{m_{\pi}^{2} e^{-m_{\pi} r}}{12 \pi r}\left[S_{12}(\hat{r})\left(1+\frac{3}{m_{\pi} r}+\frac{3}{\left(m_{\pi} r\right)^{2}}\right)+\vec{\sigma}_{1} \cdot \vec{\sigma}_{2}\right]-\frac{1}{3} \vec{\sigma}_{1} \cdot \vec{\sigma}_{2} \delta^{3}(\vec{r})\right\} .
\end{aligned}
$$

Here $\boldsymbol{\tau}$ are Pauli matrices in isospin space, $m_{\pi}$ is the pion mass, $f_{\pi}$ is the pion decay constant, $g_{A}$ is the nucleon axial charge, and $S_{12}(\hat{r})$ is the tensor operator for spin- $1 / 2$ particles defined in (3)). Recent reviews of literature relating to chiral effective field theory can be found in [47, 48]. This tensor interaction has some general similarities to that of the test potential considered here. However the divergent short-distance behavior of the one-pion exchange potential and other short-distance nucleon-nucleon interactions means that the lattice spacing plays an essential role in regulating ultraviolet divergences. The hard spherical wall method we have presented should therefore be useful in determining the underlying physics of a given lattice action. The singular short-distance interactions are likely to cause larger breaking of rotational invariance than that found for the bounded test potential. In such cases it may be useful to include higher-order derivative interactions specifically designed to cancel lattice artifacts. 


\section{Acknowledgements}

Partial financial support from the Deutsche Forschungsgemeinschaft (SFB/TR 16), Helmholtz Association (contract number VH-NG-222), and U.S. Department of Energy (DEFG02-03ER41260) are gratefully acknowledged. This research is part of the EU Integrated Infrastructure Initiative in Hadron Physics under contract number RII3-CT-2004-506078.

[1] H. M. Müller, S. E. Koonin, R. Seki, and U. van Kolck, Phys. Rev. C61, 044320 (2000), nucl-th/9910038.

[2] T. Abe, R. Seki, and A. N. Kocharian, Phys. Rev. C70, 014315 (2004), erratum-ibid. C71 (2005) 059902, nucl-th/0312125.

[3] S. Chandrasekharan, M. Pepe, F. D. Steffen, and U. J. Wiese, Nucl. Phys. Proc. Suppl. 129, 507 (2004), hep-lat/0309093.

[4] S. Chandrasekharan, M. Pepe, F. D. Steffen, and U. J. Wiese, JHEP 12, 035 (2003), heplat/0306020.

[5] D. Lee, B. Borasoy, and T. Schäfer, Phys. Rev. C70, 014007 (2004), nucl-th/0402072.

[6] D. Lee and T. Schäfer, Phys. Rev. C72, 024006 (2005), nucl-th/0412002.

[7] M. Hamilton, I. Lynch, and D. Lee, Phys. Rev. C71, 044005 (2005), nucl-th/0412014.

[8] R. Seki and U. van Kolck, Phys. Rev. C73, 044006 (2006), nucl-th/0509094.

[9] D. Lee and T. Schäfer, Phys. Rev. C73, 015201 (2006), nucl-th/0509017.

[10] D. Lee and T. Schäfer, Phys. Rev. C73, 015202 (2006), nucl-th/0509018.

[11] B. Borasoy, H. Krebs, D. Lee, and U.-G. Meißner, Nucl. Phys. A768, 179 (2006), nuclth/0510047.

[12] F. de Soto and J. Carbonell (2006), hep-lat/0610040.

[13] B. Borasoy, E. Epelbaum, H. Krebs, D. Lee, and U.-G. Meißner, Eur. Phys. J. A31, 105 (2007), nucl-th/0611087.

[14] D. Lee and R. Thomson, Phys. Rev. C75, 064003 (2007), nucl-th/0701048.

[15] J.-W. Chen and D. B. Kaplan, Phys. Rev. Lett. 92, 257002 (2004), hep-lat/0308016.

[16] M. Wingate (2005), cond-mat/0502372.

[17] A. Bulgac, J. E. Drut, and P. Magierski, Phys. Rev. Lett. 96, 090404 (2006), cond- 
mat/0505374.

[18] D. Lee, Phys. Rev. B73, 115112 (2006), cond-mat/0511332.

[19] E. Burovski, N. Prokofev, B. Svistunov, and M. Troyer, Phys. Rev. Lett. 96, 160402 (2006), cond-mat/0602224.

[20] E. Burovski, N. Prokofev, B. Svistunov, and M. Troyer, New J. Phys. 8, 153 (2006), condmat/0605350.

[21] D. Lee, Phys. Rev. B75, 134502 (2007), cond-mat/0606706.

[22] M. Lüscher, Commun. Math. Phys. 104, 177 (1986).

[23] M. Lüscher, Commun. Math. Phys. 105, 153 (1986).

[24] M. Lüscher, Nucl. Phys. B354, 531 (1991).

[25] X. Li and C. Liu, Phys. Lett. B587, 100 (2004), hep-lat/0311035.

[26] X. Feng, X. Li, and C. Liu, Phys. Rev. D70, 014505 (2004), hep-lat/0404001.

[27] S. R. Beane, P. F. Bedaque, A. Parreno, and M. J. Savage, Phys. Lett. B585, 106 (2004), hep-lat/0312004.

[28] K. Rummukainen and S. A. Gottlieb, Nucl. Phys. B450, 397 (1995), hep-lat/9503028.

[29] C. H. Kim, C. T. Sachrajda, and S. R. Sharpe, Nucl. Phys. B727, 218 (2005), hep-lat/0507006.

[30] I. Sato and P. F. Bedaque (2007), hep-lat/0702021.

[31] S. Sasaki and T. Yamazaki, Phys. Rev. D74, 114507 (2006), hep-lat/0610081.

[32] S. R. Beane, W. Detmold, and M. J. Savage (2007), arXiv:0707.1670 [hep-lat].

[33] R. C. Johnson, Phys. Lett. B114, 147 (1982).

[34] C. Lanczos, J. Res. Nat. Bur. Stand. 45, 255 (1950).

[35] H. P. Stapp, T. J. Ypsilantis, and N. Metropolis, Phys. Rev. 105, 302 (1957).

[36] J. M. Blatt and L. C. Biedenharn, Phys. Rev. 86, 399 (1952).

[37] J. M. Blatt and L. C. Biedenharn, Rev. Mod. Phys. 24, 258 (1952).

[38] C. A. Regal, C. Ticknor, J. L. Bohn, and D. S. Jin, Phys. Rev. Lett. 90, 053201 (2003), cond-mat/0209071v1.

[39] J. Zhang, E. G. M. van Kempen, T. Bourdel, L. Khaykovich, J. Cubizolles, F. Chevy, M. Teichmann, L. Tarruell, S. J. J. M. F. Kokkelmans, and C. Salomon, Phys. Rev. A 70, 030702(R) (2004).

[40] C. H. Schunck, M. W. Zwierlein, C. A. Stan, S. M. F. Raupach, W. Ketterle, A. Simoni, E. Tiesinga, C. J. Williams, and P. S. Julienne, Phys. Rev. A 71, 045601 (2005), cond- 
mat/0407373.

[41] J. P. Gaebler, J. T. Stewart, J. L. Bohn, and D. S. Jin (2007), cond-mat/0703087.

[42] J. G. E. Harris, S. V. Nguyen, S. C. Doret, W. Ketterle, and J. M. Doyle (2007), arXiv.org:0705.0713.

[43] J. Doyle, B. Friedrich, R. V. Krems, and F. Masnou-Seeuws, Eur. Phys. J. D31, 149 (2004), physics/0505201.

[44] K. Gunter, T. Stoferle, H. Moritz, M. Kohl, and T. Esslinger, Phys. Rev. Lett. 95, 230401 (2005), cond-mat/0507632.

[45] A. Micheli, G. K. Brennen, and P. Zoller, Nat. Phys. 2, 341 (2006), quant-ph/0512222v2.

[46] G. K. Brennen, A. Micheli, and P. Zoller, New J. Phys. 9, 138 (2007), quant-ph/0612180.

[47] P. F. Bedaque and U. van Kolck, Ann. Rev. Nucl. Part. Sci. 52, 339 (2002), nucl-th/0203055.

[48] E. Epelbaum, Prog. Part. Nucl. Phys. 57, 654 (2006), nucl-th/0509032. 